\title{
Awareness of the COVID-19 Outbreak and Resultant Depressive Tendencies in Patients with Severe Alzheimer's Disease
}

\author{
Akito Tsugawa, Shu Sakurai, Yuta Inagawa, Daisuke Hirose, Yoshitsugu Kaneko, Yusuke Ogawa, \\ Shuntaro Serisawa, Naoto Takenoshita, Hirofumi Sakurai, Hidekazu Kanetaka, Kentaro Hirao \\ and Soichiro Shimizu*
}

Accepted 11 August 2020

\begin{abstract}
The ongoing coronavirus disease 2019 (COVID-19) pandemic has substantially affected patients with dementia and their caregivers. However, we found not all Alzheimer's disease (AD) patients were afraid of COVID-19 infection. Therefore, we investigated the association between rate of awareness of COVID-19 and depressive tendency in AD. 126 consecutive outpatients with $\mathrm{AD}$ were enrolled in this study from May 25, on the day when the declaration of emergency was lifted in Japan, through June 30, 2020. In addition to routine psychological tests, the participants were asked the following two questions: "Do you know COVID-19?" and "Why are you wearing a face mask?". Moderate to severe AD patients were found to have a low COVID-19 recognition rate and did not fully understand why they were wearing face masks. In addition, because they did not understand the seriousness of the COVID-19 outbreak, their Geriatric Depression Scale scores were also substantially lower. These results may appear to simply indicate that people with severe dementia are unaware of current events. However, these results provide insights into how to care for patients with dementia and how to allocate the time and support of our limited staff during the COVID-19 outbreak.
\end{abstract}

Keyword: Alzheimer's disease, COVID-19, dementia, dementia care

The ongoing coronavirus disease 2019 (COVID19) pandemic has substantially affected patients with dementia and their caregivers. Owing to the restrictive measures taken worldwide to block the spread of COVID-19 outbreaks (including the declaration of a state of emergency in Japan), patients with dementia and their caregivers have not been able to receive the usual support and care. Therefore, this is expected to lead to adverse effects on the patients and their caregivers, and many investigators have warned about the

\footnotetext{
*Correspondence to: Soichiro Shimizu, MD, Department of Geriatric Medicine, Tokyo Medical University, 6-7-1 Nishishinjuku, Shinjuku-ku, Tokyo 160-0023, Japan. Tel.: +81 33342 6111; Fax: +81 33342 2305; E-mail: soichiro@tokyo-med.ac.jp.
}

risks [1-3]. In fact, many scheduled appointments for routine outpatients' examinations and care services have been canceled and postponed owing to the COVID-19 outbreak. When dementia outpatients came to our clinic for their postponed outpatient appointments after the state of emergency declaration was lifted, we found that some patients were afraid of COVID-19 infection whereas some were not. We noticed that patients with severe dementia tended to be less susceptible to COVID-19 than patients with mild dementia in the daily clinical setting. Based on the hypothesis that patients with severe dementia tend to be unaware of the COVID19 outbreak and hence may be less depressed, we compared the rate of recognition of the COVID-19 
Table 1

Awareness of COVID-19 and mental status in Alzheimer's disease

\begin{tabular}{lcc}
\hline & $\begin{array}{c}\text { Mild AD } \\
\text { MMSE } \geq 22\end{array}$ & $\begin{array}{c}\text { Moderate to Severe AD } \\
\text { MMSE }<22\end{array}$ \\
\hline Sex (male/female) & $15 / 36$ & $20 / 55$ \\
Age (y) & $80.98 \pm 5.30$ & $83.27 \pm 5.32$ \\
Years of education & $12.61 \pm 2.62$ & $12.29 \pm 2.48$ \\
MMSE score & $23.59 \pm 1.40$ & $16.53 \pm 4.06^{*}$ \\
GDS-S score & $5.73 \pm 3.30$ & $2.54 \pm 2.22^{*}$ \\
Rate of patients aware of COVID-19 & $81 \%$ & $31 \% *$ \\
Rate of patients understanding the & $78 \%$ & $24 \% *$ \\
reason for wearing a mask & & \\
\hline
\end{tabular}

AD, Alzheimer's disease; MMSE, Mini-Mental State Examination; GDS-S, Geriatric Depression Scale-Short Version. * $p<0.0001$ versus mild AD.

outbreak and resultant depressive tendencies between patients with mild dementia and those with severe dementia.

In this study, patients with Alzheimer's disease (AD) [4] are included in this study because the depressive tendency depends on the cause of dementia. A total of 126 consecutive outpatients with AD from the Memory Disorder Clinic at the Department of Geriatric Medicine, Tokyo Medical University, were enrolled in this study from May 25, on the day when the declaration of emergency was lifted, through June 30, 2020. All procedures involving human participants were in accordance with the ethical standards of the institutional research committee and with the 1964 Helsinki declaration and its later amendments or comparable ethical standards. Informed consent was obtained from all participants included in the study or their family members.

In addition to the Mini-Mental State Examination (MMSE) [5] and Geriatric Depression Scale ${ }^{-}$ Short Version $\left(\mathrm{GDS}^{-} \mathrm{S}\right)$ [6] performed as routine psychological tests, the participants were asked the following two questions: "Do you know COVID19?" and "Why are you wearing a face mask?". The patients were divided into the mild AD group (MMSE score $\geq 21, n=51$ ) and the moderate to severe AD group (MMSE score $<21, n=75$ ), and the results of the neuropsychological tests and the two questions were compared. During the state of emergency, none of the patients had received a diagnosis of COVID-19 or had required any treatment changes or additional treatments owing to behavioral and psychological symptoms of dementia. Consistent with our hypothesis, AD patients with moderate to severe cognitive impairment were found to have a low COVID-19 recognition rate and did not fully understand why they were wearing face masks. In addition, because they did not understand the seriousness of the COVID-19 outbreak, their GDS scores were also substantially lower (Table 1).

These results may appear to simply indicate that people with severe dementia are unaware of current events. However, these results provide us with insights into how to care for patients with dementia and how to efficiently use of the time and support of our limited staff during the COVID-19 outbreak. A previous study demonstrated that the COVID19 outbreak adversely affected not only cognition and neuropsychiatric symptoms but also the functional independence of patients with dementia [1]. Therefore, our results indicate that for patients with moderate to severe cognitive impairment, it may be useful to prioritize prevention of their cognitive decline and maintenance of their functional independence, whereas for those with mild cognitive impairment, it may be useful to prioritize reducing psychological stress and preventing neuropsychiatric symptoms, such as depression. Moreover, when explaining the necessity of wearing a face mask to patients with moderate to severe dementia, it is necessary to persuade them to wear a mask, as they do not understand COVID-19. Not only because they are unaware of COVID-19 that moderate to severe dementia patients are less prone to depression, but also it should be remembered that their caregivers have made great efforts trying to prevent patients from becoming depressed. Therefore, it will be important to also investigate the depressive tendencies of caregivers during the COVID-19 outbreak.

Although these findings are limited because they were obtained from a single memory clinic and small sample size, our results provide suggestions as to how to care for patients with dementia during the COVID19 outbreak. This study provides insights into ways of caring for people with dementia while the COVID19 outbreak continues, as we must work to provide 
appropriate care to old patients with dementia during all situations.

\section{ACKNOWLEDGMENTS}

We are grateful to the medical editors of the Department of International Medical Communications of Tokyo Medical University for reviewing the manuscript.

Authors' disclosures available online (https:// www.j-alz.com/manuscript-disclosures/20-0832r1).

\section{REFERENCES}

[1] Canevelli M, Valletta M, Blasi MT, Remoli G, Sarti G, Nuti F, Sciancalepore F, Ruberti E, Cesari M, Bruno G (2020) Facing dementia during the COVID-19 outbreak. J Am Geriatr Soc 68, 1673-1676.
[2] Korczyn AD (2020) Dementia in the COVID-19 period. $J$ Alzheimers Dis 75, 1071-1072.

[3] Wang H, Li T, Barbarino P, Gauthier S, Brodaty H, Molinuevo JL, Xie H, Sun Y, Yu E, Tang Y, Weidner W, Yu X (2020) Dementia care during COVID-19. Lancet 395, 1190-1191.

[4] McKhann GM, Knopman DS, Chertkow H, Hyman BT, Jack CR Jr, Kawas CH, Klunk WE, Koroshetz WJ, Manly JJ, Mayeux R, Mohs RC, Morris JC, Rossor MN, Scheltens P, Carrillo MC, Thies B, Weintraub S, Phelps $\mathrm{CH}$ (2011) The diagnosis of dementia due to Alzheimer's disease: Recommendations from the National Institute on Aging-Alzheimer's Association workgroups on diagnostic guidelines for Alzheimer's disease. Alzheimers Dement 7, 263-269.

[5] Folstein MF, Folstein SE, McHugh PR (1975) "Mini-mental state". A practical method for grading the cognitive state of patients for the clinician. J Psychiatr Res 12, 189-198.

[6] Sheikh JI, Yesavage JA (1986) Geriatic Depression Scale (GDS). Recent evidence and development of a shorter version. Clin Geron 5, 165-173. 